\title{
Breast Cancer Rehabilitation
}

National Cancer Institute

\section{Source}

National Cancer Institute. Breast Cancer Rehabilitation. NCI Thesaurus. Code C16052.

Any intervention to help breast cancer patients overcome the physical or psychological

effects of their disease or its treatment. 